\title{
Digitalisierte Psychotherapie: Die Frage nach dem "Wie"
}

\author{
Harald Baumeister $^{\mathrm{a}} \quad$ David Daniel Ebert ${ }^{\mathrm{b}} \quad$ Steffen Moritz ${ }^{\mathrm{c}}$ \\ ${ }^{a}$ Abteilung Klinische Psychologie und Psychotherapie, Institut für Psychologie und Pädagogik, Universität Ulm, \\ Ulm, Deutschland; b Faculty of Behavioural and Movement Sciences, Clinical, Neuro- \& Developmental Psychology, \\ Amsterdam, The Netherlands; ${ }^{C}$ Klinik für Psychiatrie und Psychotherapie des Universitätsklinikums Hamburg \\ Eppendorf, Hamburg, Deutschland
}

Digitalisierte Psychotherapie umfasst ein breites Spektrum von Anwendungsfeldern, von denen noch vor 10-20 Jahren ausgegangen wurde, dass sie nicht wirksam sein können wegen nicht ausreichender Therapiebeziehung, geringer Individualisierbarbarkeit und fehlender Differenziertheit. Aktuelle Übersichtsarbeiten verdeutlichen, dass eine sehr gute Evidenz zu mittlerweile sehr vielen Störungsbereichen in Bezug auf die Wirksamkeit Internet- und Mobile-basierter Interventionen (IMI) vorliegt (Übersicht siehe [Ebert et al., 2018]) und randomisiert-kontrollierte Studien zum direkten Vergleich therapeutisch begleiteter IMI mit der jeweilig etablierten Vor-Ort-Psychotherapie im Mittel keine Wirksamkeitsunterschiede feststellen können [Carlbring et al., 2018]. Dabei erweisen sich IMI nicht nur als effektiv, sondern auch als kosteneffektiv (z.B. [Paganini et al., 2018]) bei nach aktueller Studienlage anzunehmender Patientensicherheit (z.B. [Ebert et al., 2016; Karyotaki et al., 2018]). Für die letzte Aussage muss einschränkend erwähnt werden, dass die Evidenz in Bezug auf IMI und Vor-Ort-Psychotherapie nach wie vor unzureichend ist. Wirksamkeitsnachweise finden sich gleichermaßen für Videokonferenz-basierte Psychotherapie [Berryhill et al., 2019a; Berryhill et al., 2019b] und Virtual-Reality-gestützte Psychotherapie [Valmaggia et al., 2016] sowie erste Hinweise, dass auch Chatbots, d.h. automatisierte Sprachassistenten, psychische Belastungen positiv beeinflussen können [Bendig et al., 2019]. Die Frage, "ob" digitalisierte Psychotherapie wirksam ist, kann entsprechend als belegt betrachtet werden, auch wenn diese Erkenntnis aus berufspolitischen Gründen wiederholt in Zweifel gezogen worden ist.

Ob diese Form von Interventionsmöglichkeiten zur Behandlung psychischer Störungen "Psychotherapie" genannt werden sollte, ist eine zweite berufspolitisch häufig diskutierte Frage. Psychotherapie wird definiert als "gezielte, professionelle Behandlung psychischer Störungen und/oder psychisch bedingter körperlicher Störungen mit psychologischen Mitteln" [Wirtz, 2019]. Der wissenschaftliche Beirat unterteilt weiter in Psychotherapieverfahren, Psychotherapiemethoden und Psychotherapietechniken [WBP, 2019]. Bis zu diesem Differenzierungsgrad erfüllt eine Vielzahl der digital dargebotenen Behandlungsvarianten die Kriterien für "Psychotherapie". Aus unserer Sicht benötigt es einer Erweiterung des Differenzierungsgrades nach Therapiesetting, welches jenseits von Einzel- und Gruppen-

\section{KARGER}

(C) 2019 S. Karger AG, Basel
Prof. Dr. Harald Baumeister, Dipl. Psych., PP 
psychotherapie eine Differenzierung nach vor Ort und fernkommunikativ dargebotener Psychotherapie erlaubt. Und abschließend stellt sich vor dem Hintergrund von ersten verfügbaren automatischen Sprachassistenten (Chatbots) zur Unterstützung bei psychischen Belastungen [Bendig et al., 2019] die Frage, ob Psychotherapie über das Verfahren, die Methodik und die Technik oder, wie ebenfalls häufiger vorgebracht, über den unmittelbaren menschlichen Kontakt im Zusammenspiel mit den genannten Kriterien zu definieren ist.

Wie auch immer digital gestützte Therapieverfahren final bezeichnet werden, unstrittig ist mittlerweile, dass sie bei einer Subgruppe von Betroffenen wirksam sind und entsprechend ihren Platz in der Gesundheitsversorgung erhalten sollten. Hierbei rückt die Frage, wie das Potential digitalisierter Psychotherapie bestmöglich genutzt werden kann, in den Vordergrund. Das vorliegende Schwerpunktheft deckt in diesem Kontext drei zentrale Themenbereiche ab:

1 Wirkfaktoren und Änderungsmechanismen

2 Implementierungsmodelle

3 Verbesserungspotential psychotherapeutischer Maßnahmen durch technologische Möglichkeiten

Nach der generellen Wirksamkeitsfrage kommt in der Psychotherapie, bei der zumeist eine Vielzahl verschiedener Methoden und Techniken vermischt Anwendung findet, die Frage nach der differenziellen Wirksamkeit und den spezifischen Wirkfaktoren auf. Fragen wie "Welche Bestandteile sind notwendig aber ggf. auch hinreichend, um die aufgezeigte Verbesserung bewirken zu können?", "Gibt es Umsetzungsvarianten, die zu einem unterschiedlichen Grad an Wirksamkeit beitragen?", "Sind die Verfahren gleichermaßen wirksam bei verschiedenen Subgruppen von Betroffenen?" stehen derzeit im Fokus der Forschung. So zeigt sich z.B. das therapeutisch begleitete IMI wirksamer sind als therapeutisch unbegleitete [Baumeister et al., 2014], technische Ansätze zur Steigerung der Interventionsadhärenz voraussichtlich zur Wirksamkeitssteigerung beitragen [Alkhaldi et al., 2016; Baumeister et al., im Druck], das zugrundeliegende Therapieverfahren aber ggf. eine nachrangige Bedeutung bzgl. der differentiellen Wirksamkeit aufweist [Domhardt et al., 2019] und transdiagnostische Ansätze zu vergleichbaren Effekten führen könnten wie störungsspezifisch entwickelte Interventionen [Domhardt et al., 2019].

Im vorliegenden Schwerpunktheft befassen sich Probst und Kollegen mit der Frage des Zusammenhangs der therapeutischen Allianz mit dem Therapieerfolg. In Bezug auf die differentielle Wirksamkeit befassen sich Miegel und Kollegen mit der Frage, ob eine für depressive Beschwerden entwickelte Online-Interven- tion bei Menschen mit Schmerzsyndromen und depressiver Belastung sowohl die depressive Symptomatik als auch die Schmerzproblematik lindern kann. Dies stellt ein Beispiel differentieller Wirksamkeitsforschung dar, die sowohl die Frage berührt, ob Online-Interventionen transdiagnostische Effekte aufweisen können, als auch die Frage nach der Generalisierbarkeit der Wirksamkeitsnachweise über verschiedene Subpopulationen hinweg. Hierbei besteht weiterhin ein hoher Forschungsbedarf insbesondere in Bezug auf schwer erreichbare Menschen mit psychischen Störungen, die Versorgung psychischer Störungen im Kontext kultureller und sprachlicher Barrieren [Spanhel et al., 2019] sowie psychische Störungen im Kontext komplexer Behandlungsfälle, wie dies z.B. bei Menschen mit körperlichen Erkrankungen und psychischen Störungen der Fall ist [Bendig et al., 2018]. Derzeit befindet sich eine Vielzahl an Studien in Arbeit, die einerseits in sogenannten "dismantling und additive studies" [Papa und Follette, 2015; Domhardt et al., 2019] die wirksamen Bestandteile von IMI untersuchen und andererseits die differenzielle Wirksamkeit bestehender IMI in verschiedenen Populationen und Implementierungsvarianten erforschen.

In Bezug auf Implementierungsvarianten bieten zwei weitere Beiträge des Themenheftes wissenschaftliche Erkenntnisse zu der überaus bedeutsamen Frage, wie digitale Psychotherapie bestmöglich implementiert werden sollten. Hierbei geht es zum einen bei Titzler et al. um den Bereich der verzahnten Psychotherapie als Möglichkeit die Vor-Ort-Psychotherapie mit digitalen Behandlungsmöglichkeiten $\mathrm{zu}$ verschränken und $\mathrm{zu}$ augmentieren. Verzahnte Psychotherapie stellt eine sehr naheliegende Implementierungsvariante digitalisierter Behandlungsmöglichkeiten dar, wurde bislang jedoch im Vergleich zur Fülle an Studien zu für sich stehenden IMI noch selten wissenschaftlich fundiert untersucht [Baumeister et al., 2018; Ebert et al., 2018; Erbe et al., 2017]. Zum anderen fokussieren Zagorscak et al. auf die methodische Frage nach dem Zusammenhang zwischen der Rekrutierungsstrategie in klinischen Studien, der daraus resultierenden Teilnehmerpopulation und damit verbundenen potentiell differentiellen Effekten. Übergeordnet befasst sich dieser Artikel mit der Frage, wen man mit IMI-Angeboten erreicht, welche bislang unzureichend versorgten Personengruppen man mit gezielten, aktiven Disseminationsstrategien erreichen könnte und wie sich dies jeweils auf die Wirksamkeit verschiedener Interventionen auswirkt. Im Bereich der Implementierungsforschung befinden wir uns in einer Phase, in der wissenschaftlich überprüfte, aber leider auch nicht überprüfte Interventionen Betroffenen zur Verfügung gestellt werden. So zahlen Krankenkassen teilweise alternativmedizinische Ver- 
fahren ohne ausreichende wissenschaftliche Evidenz, aber verwehren Patienten die Erstattung von metaanalytisch gesichert wirksamen Verfahren, u.a. OnlineAngebote. Im Bereich der Online-Interventionen stehen eine begrenzte Anzahl wissenschaftlich fundierter, evaluierter und verfügbarer Interventionen einer Vielzahl ungeprüfter Gesundheits-Apps aus dem sekundären Gesundheitsmarkt der App Stores gegenüber ([Terhorst et al., 2018], www.mhad.science). Dieser Implementierungsprozess findet aktuell eher zufällig statt und folgt keinem wissenschaftlich fundierten Konzept. Jenseits von Zufall erscheinen vielmehr Interessen verschiedener Stakeholder ausschlaggebend für die Frage, wie IMI in unsere Versorgung integriert werden, was dem Ziel einer bestmöglichen Patientenversorgung sicherlich nicht durchgehend zuträglich ist und entsprechend dringend einer den Implementierungsprozess begleitenden Implementationsforschung bedarf [Baumeister, 2014].

Der dritte Teil des vorliegenden Themenheftes fokussiert auf den Bereich des Verbesserungspotentials psychotherapeutischer Maßnahmen, welches durch technologische Möglichkeiten und Neuerungen entsteht. Hierbei steht in den beiden Beiträgen insbesondere die Internet- und Mobile-basierte Psychodiagnos- tik im Vordergrund, die, wie im Beitrag von Lutz et al. aufgezeigt, ein hohes Potential zur Qualitätssicherung und Behandlungsoptimierung von Psychotherapie aufweist und durch Mobile-Sensing-Ansätze, wie im Beitrag von Messner et al. skizziert, in Zukunft eine diagnostisch weiter differenzierte Psychotherapie ermöglichen könnte. Mittels Data-Science-Ansätzen, die basierend auf Big Data und Machine-Learning-Konzepten Prädiktionsmodelle zu Behandlungserfolgsaussichten und Behandlungsverlaufswahrscheinlichkeiten bieten, werden der Psychotherapie in Zukunft Diagnostiksupportsysteme zur Verfügung stehen, die Psychotherapeuten und Psychotherapeutinnen in ihren Entscheidungsprozessen bezüglich Indikation, Differentialindikation, Prozessentscheidungen während der Psychotherapie sowie Abschluss- und Nachsorgemaßnahmen unterstützen.

Zusammenfassend bietet das vorliegende Themenheft einen Überblick zu drei derzeit bestehenden und drängenden Themenbereichen der digitalisierten Psychotherapie, deren Erforschung dazu beitragen kann, das Potential digitalisierter Behandlungsansätze im Sinne des Patientenwohls und der Patientensicherheit bestmöglich auszuschöpfen.

\section{Literatur}

Alkhaldi G, Hamilton FL, Lau R, Webster R, Michie S, Murray E. The effectiveness of prompts to promote engagement with digital interventions: a systematic review. J Med Internet Res. 2016 Jan;18(1):e6.

Baumeister H, Grässle C, Ebert DD, Krämer LV . Blended Psychotherapy - verzahnte Psychotherapie: Das Beste aus zwei Welten? PiD Psychother Dialog. 2018;19(04):33-8.

Baumeister H, Kraft R, Baumel A, Pryss R, Messner EM. Persuasive e-health design for behavior change. In: Baumeister $\mathrm{H}$, Montag C, editors. Digital phenotyping and mobile sensing: new developments in psychoinformatics. Berlin: Springer [im Druck].

Baumeister H, Reichler L, Munzinger M, Lin J. The impact of guidance on Internet-based mental health interventions- A systematic review. Internet Interv. 2014;1(4):205-15.

Baumeister $H$. Implementationsforschung in der Klinischen Psychologie, Rehabilitationspsychologie und Psychotherapie. Psychol Rundsch. 2014;65(3):150-8.

Bendig E, Bauereiß N, Ebert DD, Snoek F, Andersson G, Baumeister H. Internet-based interventions in chronic somatic disease. Dtsch Arztebl Int. 2018;115:659-65.

Bendig E, Erb B, Schulze-Thuesing L, Baumeister $\mathrm{H}$. The next generation: chatbots in clinical psychology and psychotherapy to foster mental health - a scoping review. Verhaltenstherapie. 2019, DOI: 10.1159/000501812.
Berryhill MB, Culmer N, Williams N, HalliTierney A, Betancourt A, Roberts $\mathrm{H}$, et al. Videoconferencing psychotherapy and depression: a systematic review. Telemed eHealth 2019a;25:435-446.

Berryhill MB, Halli-Tierney A, Culmer N, Williams N, Betancourt A, King M, et al. Videoconferencing psychological therapy and anxiety: a systematic review. Fam Pract. 2019 b Jan;36(1):53-63.

Carlbring P, Andersson G, Cuijpers P, Riper H, Hedman-Lagerlöf E. Internet-based vs. face-to-face cognitive behavior therapy for psychiatric and somatic disorders: an updated systematic review and meta-analysis. Cogn Behav Ther. 2018 Jan;47(1):1-18.

Domhardt M, Geßlein H, von Rezori RE, Baumeister H. Internet- and mobile-based interventions for anxiety disorders: A metaanalytic review of intervention components. Depress Anxiety. 2019 Mar;36(3):213-24.

Ebert DD, Donkin L, Andersson G, Andrews G, Berger T, Carlbring P, et al. Does Internetbased guided-self-help for depression cause harm? An individual participant data metaanalysis on deterioration rates and its moderators in randomized controlled trials. Psychol Med. 2016 Oct;46(13):2679-93.
Ebert DD, Van Daele T, Nordgreen T, Karekla $\mathrm{M}$, Compare A, Zarbo C, et al. Internet- and Mobile-based psychological interventions: applications, efficacy, and potential for improving mental health. Eur Psychol. 2018; 23(2):167-87.

Erbe D, Eichert HC, Riper H, Ebert DD. Blending face-to-face and Internet-based interventions for the treatment of mental disorders in adults: systematic review. J Med Internet Res. 2017 Sep;19(9):e306.

Karyotaki E, Kemmeren L, Riper H, Twisk J Hoogendoorn A, Kleiboer A, et al. Is selfguided internet-based cognitive behavioural therapy (iCBT) harmful? An individual participant data meta-analysis. Psychol Med. 2018 Nov;48(15):2456-66

Paganini S, Teigelkötter W, Buntrock C, Baumeister $\mathrm{H}$. Economic evaluations of internet- and mobile-based interventions for the treatment and prevention of depression: A systematic review. J Affect Disord. 2018 Jan; 225:733-55.

Papa A, Follette WC. Dismantling studies of psychotherapy. In: Cautin RL, Lilienfeld SO, editors. The encyclopedia of clinical psychology. Hoboken, NJ, USA: John Wiley \& Sons, Inc.; 2015. https://doi. org/10.1002/9781118625392.wbecp523. 
Spanhel K, Schweizer JS, Wirsching D, Lehr D, Baumeister H, Bengel J, et al. Cultural adaptation of internet interventions for refugees: results from a user experience study in Germany. Internet Interv. 2019, DOI: 10.1016/j. invent.2019.100252.

Terhorst Y, Rathner EM, Baumeister H, Sander L. «Hilfe aus dem App-Store?»: Eine systematische Übersichtsarbeit und Evaluation von Apps zur Anwendung bei Depressionen. Verhaltenstherapie. 2018;28(2):101-12.
Valmaggia LR, Latif L, Kempton MJ, Rus-Calafell M. Virtual reality in the psychological treatment for mental health problems: an systematic review of recent evidence. Psychiatry Res. 2016 Feb;236:189-95.

WBP (Wissenschaftlicher Beirat Psychotherapie). Methodenpapier des Wissenschaftlichen Beirats Psychotherapie nach $\$ 11$ PsychThG -Verfahrensregeln zur Beurteilung der wissenschaftlichen Anerkennung von Methoden und Verfahren der Psychotherapie. Abgerufen 14. August 2019, von https://www.wbpsychotherapie.de/fileadmin/user_upload/downloads/pdf-Ordner/ WBP/Methodenpapier.pdf
Wirtz MA. Psychotherapie. In: MA Wirtz (Hrsg.). Dorsch Lexikon der Psychologie (18. Auflage). Abgerufen 14. August 2019, von https://portal.hogrefe.com/dorsch/psychotherapie-1/ 